\title{
On Rainbow Connection Number of Some Graphs
}

\author{
Shalini Rajendra Babu, N. Ramya
}

\begin{abstract}
The Rainbow connection number for the following graphs, two copies of Fan graph $F_{n}$ by a path $P_{n}$, Arrow graph $A_{n}{ }^{2}$ and $K_{1, m} \Theta K_{1, n}$, Jellyfish graph and Cycle Cactus graph have been described in this paper
\end{abstract}

Keywords: Rainbow Coloring, Fan Graph, Arrow Graph, Corona $K_{1, m} \Theta K_{1, n}$, Jellyfish graph, Cycle Cactus graph.

\section{INTRODUCTION}

Finite, undirected and simple graphs are considered. An edge colored graph $\mathrm{G}$ is rainbow edge connected, if any two vertices are connected by a path whose edges have distinct colors. Thus, the following natural parameters was defined by charted et al [ 1].

Let the rainbow connection of a connection graph $\mathrm{G}$ denoted by $r_{c}(G)$, be the smallest number of colors, that are needed in order to make rainbow edge connected. Let $G$ be a nontrivial connected graph on which an edge coloring

$\mathrm{C}: \mathrm{E}(\mathrm{G}) \rightarrow\{1,2, \ldots n\}, \mathrm{n} \in \mathrm{N}$, is defined where adjacent edges may be colored the same. Rainbow connection has an interesting application for the secure transfer of classified information between agencies, while the information needs to be protected since it relates to national security, there must also be procedures that permit access between appropriate parties. [3]

Then we consider the rainbow coloring of the following graphs,

(i) Two copies of Fan graph by a Path $P_{n}$

(ii) Arrow graph $A_{n}{ }^{2}$

(iii) $K_{1, m} \Theta K_{1, n}$

(iv) Jelly Fish graph

(v) Cycle-Cactus graph

(vi) 2-Tuple graph

\section{DEFINITION}

\section{A. Fan graph}

A Fan graph $F_{m_{n} n}$ is defined as the graph $\overline{k_{m}}+\mathrm{P}_{\mathrm{n}}$, where $\overline{k_{m}}$ is the empty graph on $m$ nodes and $P_{n}$ is the path graph on $n$ nodes, when $m=1$, corresponds the usual Fan graph.

\section{B. Arrow graph}

An arrow graph $A_{n}{ }^{2}$ with width 2 and length $\mathrm{n}$ is obtained by joining a vertex $\mathrm{V}$ with superior vertices of $p_{2} \times p_{n}$ by 2 new edges from one end. [7]

\section{Corona $\mathrm{K}_{1, \mathrm{~m}} \Theta \mathrm{K}_{1, \mathrm{n}}$}

$K_{1, m} \Theta K_{1, n}$ is a tree obtained by adding $n$ pendant vertices of $K_{1, m}$.

\section{Jelly Fish graph}

The jelly fish graph $J(m, n)$ is obtained from a 4-cycle $V_{1}, V_{2}, V_{3}, V_{4}$ by joining $V_{1}$ and $V_{3}$ with an edge and appending m pendant edges to $V_{2}$ and n pendant edges to $V_{4} \cdot[6]$

\section{E. Cactus}

A cactus is a connected graph in which any two simple cycles have at most even vertex in common

\section{F. 2-Tuple graph}

Let $G=(V, E)$ be a simple graph and $G^{0}=\left(V^{0}{ }_{2} E^{0}\right)$ be another copy of graph $G$. Join each vertex $v$ of $G$ to the corresponding vertex $V^{0}$ of $G^{0}$ by an edge. The new graph thus obtained we call 2-tuple graph of $\mathrm{G}$. We denote 2-tuple graph of $G=(p, q)$ then

$$
\left|V\left(T^{2}(G)\right)\right|=2 p \text { and }\left|T^{2}(G)\right|=2 p+q .[9]
$$

\section{MAIN RESULTS}

\section{Theorem 1:}

The graph $\mathrm{G}$ is obtained by joining two copies of fan graph $F_{\mathrm{n}}$ by a path of arbitrary, whose Rainbow coloring is $n-1_{s}{ }^{\prime \prime} n^{\mathrm{I}}$ denotes number of vertices in the Fan graph.
Revised Manuscript Receiv
* Correspondence Author

Shalini Rajendra Babu*, BIHER, Chennai, India. Email: rshalini19@hotmail.com

Dr.N. Ramya, ,BIHER, Chennai, India. Email: drramyamaths@gmail.com

(C) The Authors. Published by Blue Eyes Intelligence Engineering and Sciences Publication (BEIESP). This is an open access article under the CC BY-NC-ND license (http://creativecommons.org/licenses/by-nc-nd/4.0/) 


\section{On Rainbow Connection Number of Some Graphs}

Proof:

Let $\mathrm{G}$ be the graph obtained by joining two copies of Fan graph $F_{n}$ by a path $P_{k}$ of length $k-1$. [2]. Let us denote the successive vertices of first copy of Fan graph by

$u_{1}, u_{2}, u_{3} \ldots u_{n+1}$ and the successive vertices of second copy of fan graph by $w_{1}, w_{2}, w_{n} \ldots w_{n+1}$. Let $v_{1}, v_{2}, u_{n} \ldots v_{k}$ be the path $P_{k}$ and $v_{k}=w_{\mathrm{i}}$. For $n=3, F_{\mathrm{a}}$ is cycle $C_{\mathrm{a}}$, its Rainbow coloring is discussed in [5], that the graph obtained by joining of $P_{n}$ and $K_{2}$ admits a rainbow coloring with $2 n-1$ colors. Here we consider the case for $n>3$, we define a function $f: E(G) \rightarrow\{1,2,3 \ldots n-1\}$ as follows,

Coloring must be given,

$$
\begin{aligned}
& f\left(u_{i}, u_{i+1}\right)=i_{s} 1 \leq i \leq n-2 \\
& f\left(v_{i}, u_{i}\right)=1 \text { for all } i \\
& f\left(w_{,}, w_{i+1}\right)=i, 1 \leq i \leq n-2 \\
& f\left(v_{i}, v_{i+1}\right)=i+1,1 \leq i \leq n-2 \\
& f\left(v_{i}, w\right)=n-1_{2} \text { for all } i
\end{aligned}
$$

\section{Illustrations}

Rainbow coloring of the graph obtained by joining two copies of $F_{6}$ by a path $P_{4}$ shown in figure

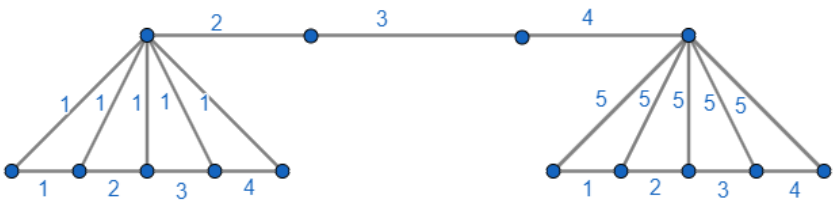

Thus, the rainbow coloring of the above graph is 5 .

Theorem 2:

An Arrow graph $A_{n}{ }^{2}$ which admits a Rainbow coloring, and whose Rainbow connection number is $n$.

Proof:

Let $A_{n}{ }^{2}$ be an Arrow graph combined by a vertex $v_{0}$ with superior vertices of $p_{2} \times p_{n}$ by 2 new edges.

Let us denote $v_{0}$ be the starting vertex. $v_{1}, v_{2} \ldots \ldots v_{n}$ be the upper vertices of Arrow graph, which has to be connected with $v_{0}$. Similarly, $w_{1}, w_{2}, \ldots, w_{n}$ be the lower vertices of an Arrow graph, which has to be connected with $v_{0}$. Here we consider the case for $n \geq 3$, we define the function

$f: E(G) \rightarrow\{1,2,3, \ldots n\}$ as follows, coloring has to be given Rainbow coloring of $A_{p}^{2}$

$$
\begin{aligned}
& f\left(v_{0}, v_{i}\right)=1 \\
& f\left(v_{i}, v_{i+1}\right)=i+1 \text { for } i \leq 1 \leq n \\
& f\left(v_{0}, w_{1}\right)=1 \\
& f\left(w_{i}, w_{i+1}\right)=i+1, \text { for } 1 \leq i \leq n \\
& f\left(w_{n}, v_{n}\right)=1
\end{aligned}
$$

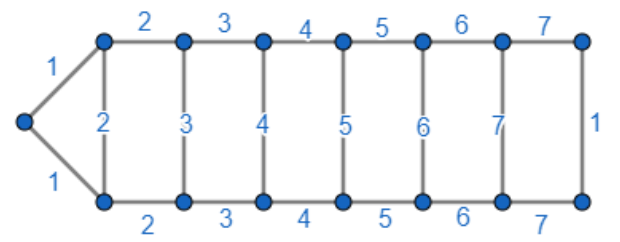

Thus, the rainbow coloring of the above graph is 7 .

\section{Theorem 3:}

The graph $K_{1, m} \Theta K_{1, n}$ for all m, n which admits Rainbow coloring, whose rainbow connection number $n$. Where ' $n$ ' represents number of edges.
Proof:

Let $v_{0}$ be the root of the tree. Let. $v_{1}, v_{2 x \ldots \ldots} v_{m}$ be the children of the root. Each subtree $v_{i}, 1 \leq i \leq m_{x}$ will have 'n' number of vertices which have $v_{i 1}, v_{i 2} \ldots \ldots v_{i n}$

leaves [4]. The vertices that acts as a leaf of the graph $K_{1 \mathrm{~m}} \Theta$ $K_{1 \text { n }}$ are colored as follows

\section{Illustration}

Case (i) when $m=n$

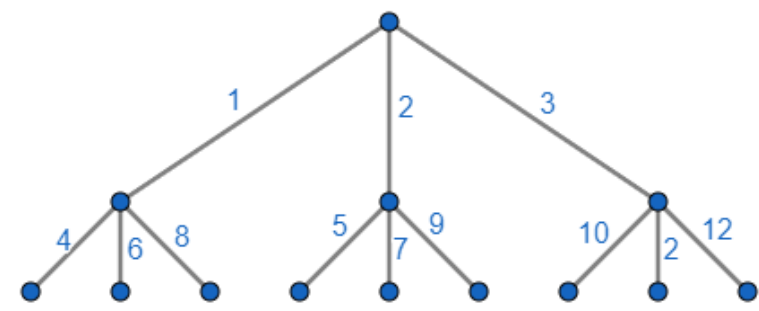

Rainbow coloring of $K_{1, a} \Theta K_{1, a}$ is 12 .

Case (ii) when $m \neq n$

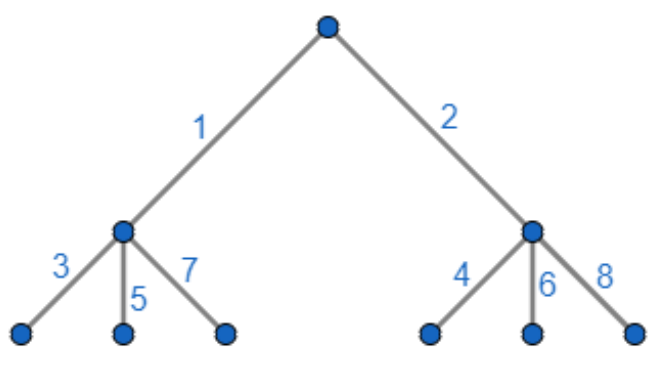

$$
K_{1,2} \Theta K_{1, a}
$$

Thus, the rainbow coloring of the above graph is 8 .

Theorem: 4

Given the graph $\mathrm{G}$ as $T^{2}\left(P_{n} \times P_{2}\right) n \geq 2$ then rainbow connection number $\operatorname{re}(G)$ is exactly 3 .

Proof:

Let $\left\{x i_{v} y i_{v} x i^{n} y i^{i}, 1 \leq i \leq n\right\}$ be the vertices

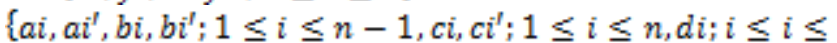
$2 n\}$

be the edges.

Construct the characteristics of $\mathrm{G}$ to integers as follows

Define

$$
\begin{gathered}
f^{*} E: \rightarrow\{1,2,3\} \\
f^{*}(a i)=1 \text { for all } i \\
f^{*}\left(a i^{\prime}\right)=1 \text { for all } i \\
f^{*}(b i)=1 \text { for all } i \\
f^{*}\left(b i^{\prime \prime}\right)=1 \text { for all } i \\
f^{*}(c i)=2 \text { for all } i \\
f^{*}(d i)=3 \text { for all } i
\end{gathered}
$$




\section{Illustration: $T^{2}\left(P_{4} \times P_{2}\right)$}

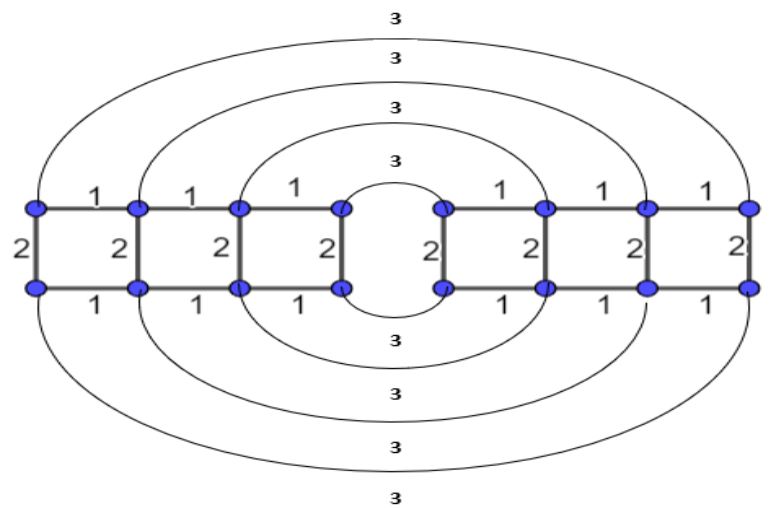

Thus the Rainbow connection number $r c(G)$ is exactly 3 as seen in (A).

It clearly shows the $\operatorname{rc}(G)$ is the minimum number of color needed to edge coloring at least one of paths in G.

\section{Theorem:5}

For every $m \geq 1, n \geq 1$ there exists a Jelly fish graph which admits a rainbow coloring with rainbow connection number is $m+n+2$.

Proof:

Let $\mathrm{G}$ be the graph with $m+n+4$ vertices and $m+n+5$ edges where $m$ represents number of pendent edges in left hand side, $\mathrm{n}$ represents its number of pendent edges in the right hand side and $E(G)=E_{1} \cup E_{2}$ where

$E_{1}=\{x u, u y, y v, v x, x y\}$

$E_{2}=\left\{\right.$ wui, vvj, $\left.1 \leq i \leq m_{v} 1 \leq j \leq m\right\}$

$u i$ 's are from left pendant edges

$v i$ 's are from right pendant edges

Labeling has to be defined as,

$$
\begin{gathered}
f^{*}: E(G) \rightarrow\{1,2, \ldots m+n+2\} \\
f^{*}(x y)=1 \\
f^{*}(x u)=1 \\
f^{*}(y u)=1 \\
f^{*}(x v)=2 \\
f^{*}(v y)=2 \\
f^{*}(\text { wui })=2+i_{s} \text { for } i=1,2, \ldots m \\
f^{*}(\text { vvj })=3+m+j, \text { for } j=0,1,2, \ldots n-1
\end{gathered}
$$

\section{Claim: $f^{*}$ is rainbow coloring}

Proof: From (i) and (vii) equations found above establishes the rainbow connection number as

$m+n+2$

\section{Illustration: $7(4,7)-$ jelly fish}

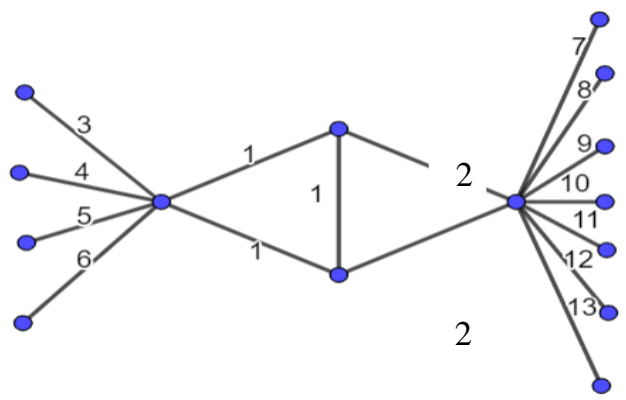

In the above graph $7(4,7)$ graph has Rainbow connection number as 13 .

\section{Theorem: 6}

A cycle-cactus $C_{k}^{(n)}$, consist of n copies of cycle $C_{k,} k \geq 3$, concatenated at exactly one vertex, which holds Rainbow connection number ' $k$ ' where ' $k$ ' is the number of vertices of the cycle.

\section{Proof:}

Let $G_{1}, G_{2}, \ldots, G_{n}$ be the copies of cycles $C_{k}$ all concatenated at exactly one vertex namely $x_{0}$.

Let $x_{0}, x_{11}, x_{12}, x_{12} x_{1 k}$ be the vertices of $G_{1}, x_{0}, x_{21}, x_{22,}, x_{2 \pi}, \ldots x_{2 k}$ be the vertices of $G_{2}$, finally let $x_{0}, x_{n 1}, x_{n 2}, x_{n 2} \ldots x_{n k}$ be the vertices of $G_{n} \times$

Let $f^{*}: E(G) \rightarrow\{1,2,3, \ldots k\}$, then the coloring has been given as follows

$$
\begin{aligned}
& f\left(x_{0}, x_{11}\right)=1 \\
& f\left(x_{0}, x_{21}\right)=1
\end{aligned}
$$

$$
\begin{gathered}
f\left(x_{n 1}, x_{n 2}\right)=2, \text { for all } \mathrm{n} \geq \mathbf{1} \\
f\left(x_{12}, x_{13}\right)=3 \\
f\left(x_{22}, x_{23}\right)=\mathbf{3}
\end{gathered}
$$

$$
\begin{gathered}
f\left(x_{n 2}, x_{n 3}\right)=3, \text { for all } \mathrm{n} \geq 1 \\
f\left(x_{1, k-1}, x_{0}\right)=k \\
f\left(x_{2, k-1}, x_{0}\right)=k \\
\cdot \\
\cdot \\
f\left(x_{n, k-1}, x_{0}\right)=k, \text { for all } \mathrm{n} \geq 1
\end{gathered}
$$




\section{On Rainbow Connection Number of Some Graphs}

Illustration: Cycle-Cactus $C_{4}{ }^{(4)}$

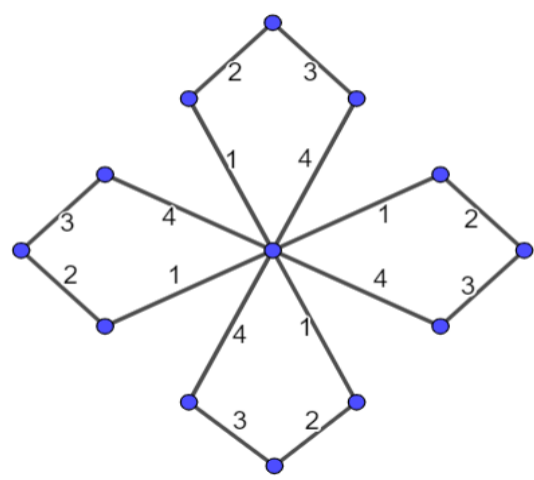

In the above graph the rainbow connection number is 4.

Cycle-Cactus $C_{5}{ }^{(4)}$

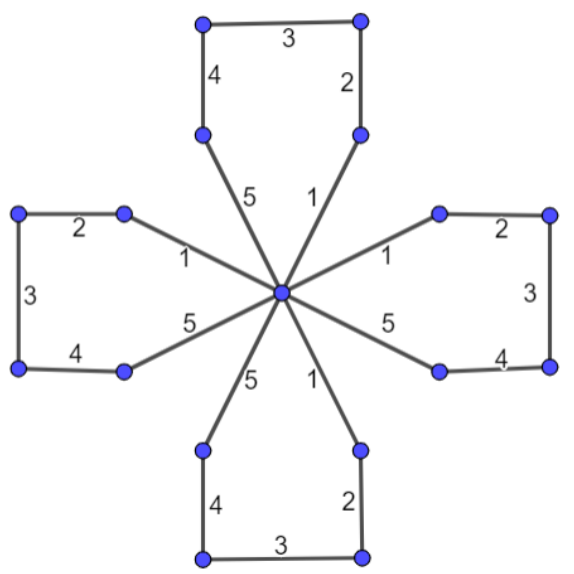

In the above graph the rainbow connection number is 5.

\section{Findings:}

\begin{tabular}{|c|c|c|}
\hline S.no & Graph & $\begin{array}{c}\text { Rainbow } \\
\text { Connection number }\end{array}$ \\
\hline 1 & $\begin{array}{l}\text { Two copies of Fan } \\
\text { graph by a path }\end{array}$ & $\begin{array}{c}n-1 \\
\text { n-number of vertices }\end{array}$ \\
\hline 2 & Arrow graph $A_{n}{ }^{2}$ & n-number of vertices \\
\hline 3 & $\begin{array}{l}\text { Corona graph } \\
K_{1 m} \otimes K_{1 n}\end{array}$ & n-number of vertices \\
\hline 4 & Jelly Fish graph & $\begin{array}{c}\mathrm{m}+\mathrm{n}+2 \\
\mathrm{~m} \text { - Pendent edges in } \\
\text { LHS } \\
\mathrm{n} \text { - Pendent edges in } \\
\text { RHS }\end{array}$ \\
\hline 5 & $\begin{array}{l}\text { Cycle-Cactus } \\
\text { graph }\end{array}$ & $\begin{array}{c}\mathrm{k} \\
\mathrm{k}-\text { number of } \\
\text { Vertices }\end{array}$ \\
\hline 6 & 2-Tuple graph & 3 \\
\hline
\end{tabular}

\section{CONCLUSION:}

It is of interest to study the connection number for various classes, after than what has been found in the literature.

\section{REFERENCES}

1. G. Chartrand, G.L.Johns, K.A. Mc Keon, P.Zhang. " Rainbow connection in graphs" Math Bohem 133 (2008), 85-98.

2. G.V.Ghodasasa, J.P Jena " Prime Cordial Labeling of some Special Graph families" Int. Journal of Mathematics and soft Computing, Vol 4 (2), 2014.

3. R.Prabha and Indra Rajasingh " Rainbow Coloring of Crown Graphs", J.Comp \& Math.Sci.Vol.3(3), 390-394(2012).

4. J.Baskar Babujee and L.Shobana, "Prime and Prime Coloring labeling for Some Special Graphs", Int. Journal of Contemp .Math Sciences, Vol.5,2010, no.47,2347-2356.

5. N.Ramya, K.Rangarajan and R.Sattanathan, " On Rainbow Coloring of Some Classes of Graphs", Int. Journal of Computer Applications, Vol.46.no 18 May 2012.

6. "Pair sum labeling of some special graphs" K.Manimekalai, K. Thirusangu International Journal of Computer Applications Vol.69.May 2013

7. "Colorful Labeling of arrow graphs and double arrow graphs".V.J.Kaneria, M.M. Jariya and H.M.Makadia Malaya Journal Of Mathematik 3(4).2015.

8. Sharon Philomena.V and K.Thirusangu "Square and Cube difference Labeling of Cycle Cactus, Special Tree and a New Key Graphs" Annals of Pure and Applied Mathematics Vol.8.2014

9. "Some Graph Operations of Even Vertex Odd Mean Labeling Graphs" M.Kannan, R.Vikrama Prasad and R.Gobi International Journal of Applied Engg. Research Vol.12.2017.

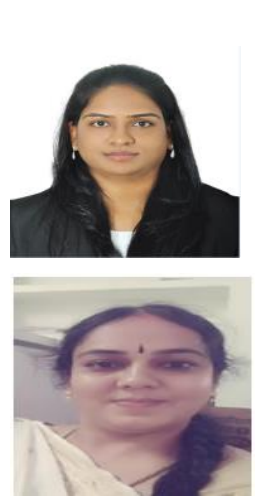

\section{AUTHORS PROFILE}

Mrs.Shalini Rajendra Babu, Pursuing Ph.D in the area of Graph theory at BIHER, Chennai. She has presented papers in Conferences and published one paper.

Dr.N. Ramya, obtained Ph.D in the field of Graph theory. She published 15 papers in National as well international journals. She is working as a professor at BIHER Chennai. 